\title{
Data Assimilation in Multiscale Chemical Transport Models
}

\author{
Lin Zhang and Adrian Sandu \\ Department of Computer Science, Virginia Polytechnic Institute and State University, \\ Blacksburg, VA 24061, US \\ \{lin83, asandu\}@vt.edu
}

\begin{abstract}
In this paper we discuss variational data assimilation using the STEM atmospheric Chemical Transport Model. STEM is a multiscale model and can perform air quality simulations and predictions over spatial and temporal scales of different orders of magnitude. To improve the accuracy of model predictions we construct a dynamic data driven application system (DDDAS) by integrating data assimilation techniques with STEM. We illustrate the improvements in STEM field predictions before and after data assimilation. We also compare three popular optimization methods for data assimilation and conclude that LBFGS method is the best for our model because it requires fewer model runs to recover optimal initial conditions.
\end{abstract}

Keywords: STEM, Chemical Transport Model, Data Assimilation.

\section{Introduction}

The development of modern industry has brought about much pollutant to the world, which has deep influence to people's life. To analyze and control the air quality, large comprehensive models are indispensable. STEM(Sulfur Transport Eulerian Model) [1] is a chemical transport model used to simulate the air quality evolutions and make predictions. A large variety of species in the air are changing at different time and space magnitude, which requires STEM to be a multiscale system to fully simulate these changes in the atmosphere.

The dynamic incorporation of additional data into an executing application is an essential DDDAS concept with wide applicability (http://www.cise.nsf.gov/dddas). In this paper we focus on data assimilation, the process in which measurements are used to constrain model predictions; the information from measurements can be used to obtain better initial conditions, better boundary conditions, enhanced emission estimates, etc. Data assimilation is essential in weather/climate analysis and forecast activities and is employed here in the context of atmospheric chemistry and transport models.

Kalman filter technique [2] gives a stochastic approach to the data assimilation problem, while variational methods (3D-Var, 4D-Var) provide an optimal control approach. Early applications of the four-dimensional variational (4D-Var) data assimilation were presented by Fisher and Lary [3] for a stratospheric photochemical box model with trajectories. Khattatov et al. [4] implemented both the 4D-Var and a 
Kalman filter method using a similar model. In the past few years variational methods have been successfully used in data assimilation for comprehensive threedimensional atmospheric chemistry models (Elbern and Schmidt [5], Errera and Fonteyn [6]). Wang et al. [7] provide a review work of data assimilation applications to atmospheric chemistry. As our STEM model is time dependent in 3D space, 4DVar is the appropriate approach to data assimilation.

The paper is organized as follows. Second section introduces the STEM Chemical Transport Model. Theory and importance of 4D-Var data assimilation are presented in the third section, followed by some results of data assimilation using the STEM in the forth section. In section five, we briefly describe L-BFGS(Limited-memory Broyden Fletcher Goldfarb Shanno method), Nonlinear Conjugate Gradients and Hessian Free Newton methods and assess their performances in the STEM model. Summary and conclusions are given in section six.

\section{The STEM Chemical Transport Model}

The STEM is a regional atmospheric Chemical Transport Model(CTM). Taking emissions, meteorology(wind, temperature, humidity, precipitation etc.) and a set of chemical initial and boundary conditions as input, it simulates the pollutants behavior in the selected domain. In the following we give the mathematical description of the Chemical Transport Model [8].

\subsection{Chemical Transport Model in STEM}

We denote $u$ the wind filed vector, $K$ the turbulent diffusivity tensor, $\rho$ the air density in molecues $/ \mathrm{cm}^{3}$. Let $V_{i}^{\text {dep }}$ be the deposition velocity of species $i, Q_{i}$ the rate of surface emissions, and $E_{i}$ the rate of elevated emissions for this species. The rate of chemical transformations $f_{i}$ depends on absolute concentration values; the rate at which mole-fraction concentrations change is then $f_{i}(\rho c) / \rho$.

Consider a domain $\Omega$ which covers a region of the atmosphere with the boundary $\partial \Omega$. At each time moment the boundary of the domain is partitioned into $\partial \Omega=\Gamma^{I N} \cup \Gamma^{O U T} \cup \Gamma^{G R}$, where $\Gamma^{G R}$ is the ground level portion of the boundary; $\Gamma^{I N}$ is the inflow part of lateral or top boundary and $\Gamma^{\text {OUT }}$ the outflow part.

The evolution of concentrations in time is described by the material balance equations

$$
\begin{gathered}
\frac{\partial c_{i}}{\partial t}=-u \cdot \nabla c_{i}+\frac{1}{\rho} \nabla \cdot\left(\rho K \nabla c_{i}\right)+\frac{1}{\rho} f_{i}(\rho c)+E_{i}, \quad t^{0} \leq t \leq T \\
c_{i}\left(t^{0}, x\right)=c_{i}^{0}(x), \\
c_{i}(t, x)=c_{i}^{I N}(t, x) \quad \text { for } \quad x \in \Gamma^{I N}, \\
K \frac{\partial c_{i}}{\partial n}=0 \quad \text { for } \quad x \in \Gamma^{O U T},
\end{gathered}
$$




$$
K \frac{\partial c_{i}}{\partial n}=V_{i}^{d e p} c_{i}-Q_{i} \quad \text { for } \quad x \in \Gamma^{G R}, \quad \text { for } \quad \text { all } \quad 1 \leq i \leq s .
$$

The system (1)-(5) builds up the forward (direct) model. To simplify the presentation, in this paper we consider the initial state $c^{0}$ as parameters of the model. It is known that this does not restrict the generality of the formulation.

An infinitesimal perturbation $\delta c^{0}$ in the parameters will result in perturbations $\delta c_{i}(t)$ of the concentration fields. These perturbations are solutions of the tangent linear model. In the direct sensitivity analysis approach we can solve the model (1)(5) together with the tangent linear model forward in time.

\subsection{Continuous Adjoint Model in STEM}

Consider a scalar response functional defined in terms of the model solution $c(t)$

$$
J\left(c^{0}\right)=\int_{t^{0}}^{T} \int_{\Omega} g(c(t, x)) d x d t
$$

The response depends implicitly on the parameters $c^{0}$ via the dependence of $c(t)$ on $c^{0}$. The continuous adjoint model is defined as the adjoint of the tangent linear model. By imposing the Lagrange identity and after a careful integration by parts one arrives at the following equations that govern the evolution of the adjoint variables:

$$
\begin{gathered}
\frac{\partial \lambda_{i}}{\partial t}=-\nabla \cdot\left(u \lambda_{i}\right)-\nabla \cdot\left(\rho K \nabla \frac{\lambda_{i}}{\rho}\right)-\left(F^{T}(\rho c) \lambda\right)_{i}-\phi_{i} \quad T \geq t \geq t^{0} \\
\lambda_{i}(T, x)=\lambda_{i}^{F}(x) \\
\lambda_{i}(t, x)=0 \quad \text { for } \quad x \in \Gamma^{I N} \\
\lambda_{i} u+\rho K \frac{\partial\left(\lambda_{i} / \rho\right)}{\partial n} \quad \text { for } x \in \Gamma^{O U T} \\
\rho K \frac{\partial\left(\lambda_{i} / \rho\right)}{\partial n}=V_{i}^{\text {dep }} \lambda_{i} \text { for } x \in \Gamma^{G R}, \text { for all } 1 \leq i \leq s
\end{gathered}
$$

Where

$$
\phi_{i}(t, x)=\frac{\partial g\left(c_{1}, \ldots, c_{n}\right)}{\partial c_{i}}(t, x), \quad \lambda_{i}^{F}(x)=0,
$$

and $\lambda_{i}(t, x)$ are the adjoint variables associated with the concentrations $c_{i}(t, x)$, $1 \leq i \leq s$. In the above $F=\partial f / \partial c$ is the Jacobian of the chemical rate function $f$. To obtain the ground boundary condition we use the fact that $u \cdot n=0$ at ground level. We refer to (7)-(11) as the (continuous) adjoint system of the tangent linear model. In the context of optimal control where the minimization of the functional (6) is required, the adjoint variables can be interpreted as Lagrange multipliers.

The adjoint system (7)-(11) depends on the states of the forward model (i.e. on the concentration fields through the nonlinear chemical term $F(\rho c)$ and possibly through the forcing term $\phi$ for nonlinear functionals. Note that the adjoint initial condition is 
posed at the final time $T$ such that the forward model must be first solved forward in time, the state $c(t, x)$ saved for all $\mathrm{t}$, then the adjoint model could be integrated backwards in time from $T$ down to $t^{0}$.

\subsection{Properties of STEM}

The model uses the SAPRC-99(Statewide Air Pollution Research Center's chemical mechanism) [9] and KPP(the Kinetic PreProcessor) [10], to determine chemical reactions. KPP implements integration of chemical mechanism using implicit Rosenbrock and Runga-Kutta method in both forward and adjoint model.

The STEM model runs multiscale simulations in both time and space. From the time aspect of view, it ranges from $10^{-6}$ seconds for fast chemical reactions to days' simulation measured in hours. Fast chemical reactions are referred to atomic level reactions, such as $\mathrm{O}, \mathrm{OH}$ radical activities, while long term simulation usually accounts for atmospheric species transportation in large range. When it comes to spatial scales, STEM is able to simulate in range measured in meters, such as emissions of pollutants like $\mathrm{NO}, \mathrm{NO}_{2}, \mathrm{CO}_{2}$, Volatile Organic Compounds (VOC) and particles from vehicles. Besides, continental scales as large as thousands of kilometers are usually used in STEM for air quality simulation. So far STEM has been employed for simulations over U.S., Asia and Europe.

For this paper, we use STEM to run on a horizontal resolution of $60 \mathrm{Km}$ by $60 \mathrm{Km}$, with 21 vertical levels defined in the Regional Atmospheric Modeling System's sigma-z coordinate system. The domain covers northeast of U.S, ranging from $68^{\circ} \mathrm{W}$ to $85^{\circ} \mathrm{W}$ and from $36^{\circ} \mathrm{N}$ to $48^{\circ} \mathrm{N}$. The simulations are carried out from $8 \mathrm{am}$ EDT to 8pm EDT on July 20, 2004, and dynamical time step is 15 minutes.

\section{4D-Var Data Assimilation in STEM}

Data assimilation is the process by which measurements are used to constrain the model predictions; the information from measurements can be used to obtain better initial conditions, better boundary conditions, enhanced emission estimates, etc. Data assimilation combines information from three different sources: the physical and chemical laws of evolution (encapsulated in the model), the reality (as captured by the observations), and the current best estimate of the distribution of tracers in the atmosphere.

In this paper we focus on obtaining optimized initial conditions which are essential in forward model integration. 4D-Var data assimilation can be used to STEM and is expected to improve air quality forecasting. In practice, directly measuring the parameters of the atmospheric conditions in large range is difficult because of sampling, technical and resource requirements. And due to the complexity of the chemical transport model, the number of possible state variables is extremely large. However, data acquisition for field and parameter estimates via data assimilation is feasible, even though enough observations are still need to fulfill data assimilation.

Figure 1 shows the employment of data assimilation in multiscale systems. The atmospheric chemical and transport processes take place in a variable range of time and space. The observations for data assimilation can come from local stations, planes, and satellites, measured from second to weeks in time and from $\mathrm{nm}$ to $\mathrm{km}$ in space. Data assimilation helps models to improve environmental policy decisions. 


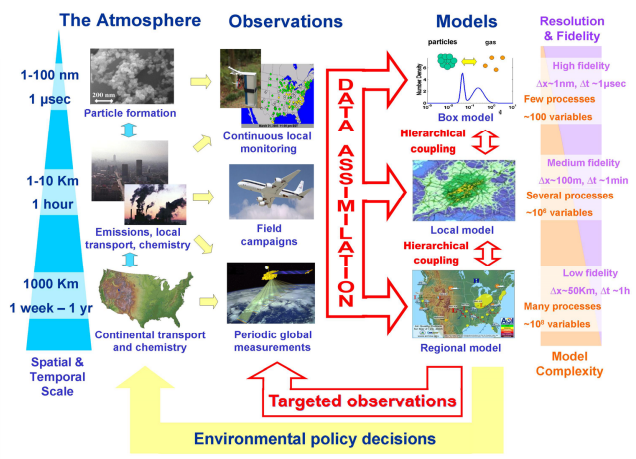

Fig. 1. Atmospheric Chemical and Transport Processes take place at multiple temporal and spatial scales

We applied 4D-Var data assimilation to obtain the optimal initial conditions using STEM by minimizing a cost function that measures the misfit between model predictions and observations, as well as the deviation of the solution from the background state. The cost function is formulated as

$$
\min J\left(c^{0}\right)=\frac{1}{2}\left(c^{0}-c^{B}\right)^{T} B^{-1}\left(c^{0}-c^{B}\right)+\frac{1}{2} \sum_{k=0}^{N}\left(H_{k} c^{k}-c_{o b s}^{k}\right)^{T} R_{k}^{-1}\left(H_{k} c^{k}-c_{o b s}^{k}\right)
$$

and our goal is to minimize the cost function $J$. In the above formula, $c^{B}$ represents the 'a priori' estimate (background) of the initial values and $B$ is associated covariance matrix of the estimated background error. $H_{K}$ is an observation operator and $c_{o b s}^{k}$ is the real observations depending on time $k$. The covariance matrix $R_{k}^{-1}$ accounts for observations and representativeness errors.

The 4D-Var data assimilation is implemented by an iterative optimization procedure: each iteration requires STEM to run a forward integration to obtain the value of the cost function and an adjoint model to evaluate the gradient. Since model states are as high as $10^{6}$ in our air quality simulation problem, it is prohibitive to evaluate Hessian of the cost function. Therefore, we choose three optimization methods that only require the values and the gradients of the cost function.

\section{Results for Data Assimilation}

We performed data assimilation to optimize initial conditions. The simulation interval is from 8am EDT to 8pm EDT on July 20, 2004. Figure 2 shows the simulation domain and three selected AIRNOW stations. AIRNOW stations provide hourly observations of ground level ozone throughout the entire month of July 2004. To show the change of ozone in this time interval at one location, we choose three out of all AirNow stations. The ozone time series at these three stations are illustrated in Figure 3. From the figure, we can find that the assimilated lines are closer to observations than non-assimilated lines, which indicates the improvement in model predictions after data assimilation. This is also confirmed by the scatter and 
quantile-quantile plots of Figure 4, which indicate that the correlation coefficient between model predictions and observations increases considerably from $\mathrm{R}^{2}=0.15$ for the original model to $\mathrm{R}^{2}=0.68$ after assimilation.

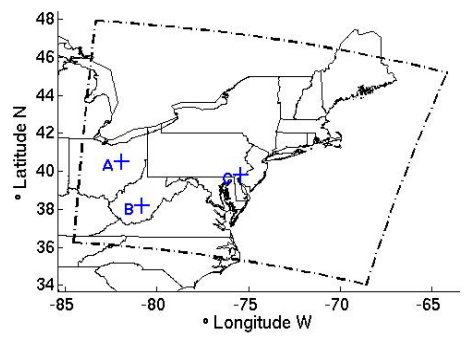

Fig. 2. Three selected stations where $\mathrm{O} 3$ time series are considered

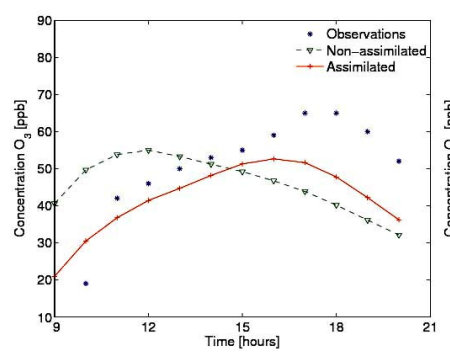

(a) station $\mathrm{A}$

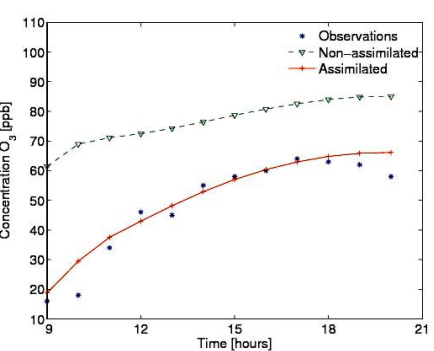

(b) station $\mathrm{B}$

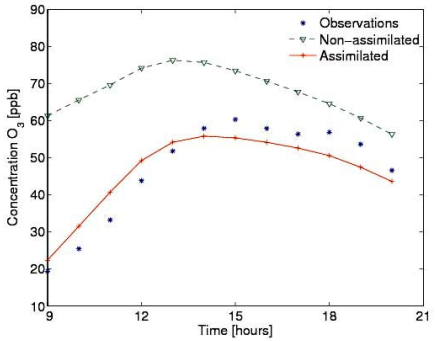

(c) station $\mathrm{C}$

Fig. 3. Time series of ozone concentrations

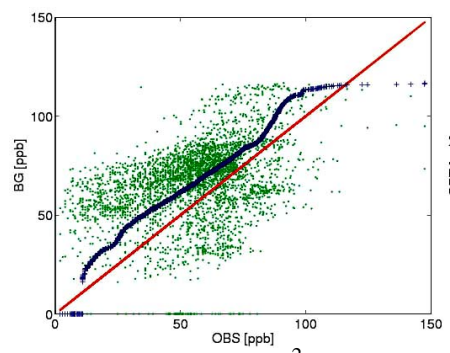

(a) Original $\left(\mathrm{R}^{2}=0.15\right)$

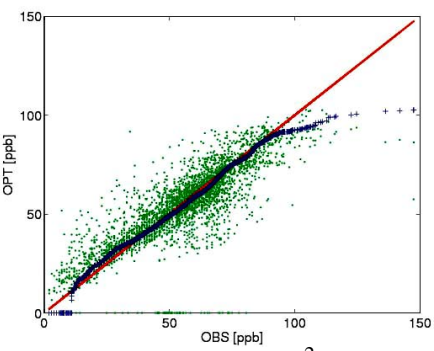

(b) Assimilated $\left(\mathrm{R}^{2}=0.68\right)$

Fig. 4. Scatter plot and quantile-quantile plot of model-observations agreement 


\section{Assessment of Three Optimization Methods}

We applied three optimization methods: L-BFGS, Nonlinear Conjugate Gradient and Hessian Free Newton for data assimilation in STEM and assess the performances of them. The principle of L-BFGS [11] is to approximate Hessian matrix G by a symmetric positive definite matrix $\mathrm{H}$, and update $\mathrm{H}$ at each step using the information from the $m$ most recent iterations. Nonlinear Conjugate Gradient method is an iterative method and generates a set of search directions $\left\{p_{0}, p_{1}, \ldots, p_{m}\right\}$ conjugating with each other for $i \neq j$. The Fletcher-Reeves Conjugate Gradient method is used in this paper. Hessian Free Newton is an inexact Newton method in which the Hessian matrix is not available, and we use automatic differentiation or finite differences to approximate the products of the Hessian times a vector [12].

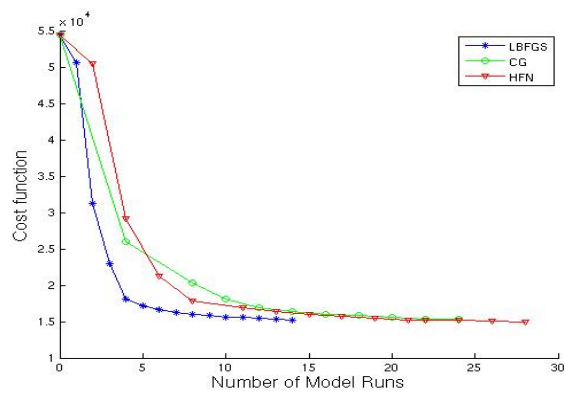

Fig. 5. Decrease of the cost function vs. the number of model runs for three methods

These methods are tested respectively to optimize initial concentrations. All the optimizations start at the same cost function of around 54800 and converge at about 16000 within 15 iterations, which proves that they are all able to solve data assimilation in STEM model. The difference lies in the number of model runs when they converge. For every model run, the STEM calls forward model and adjoint model to evaluate value and gradient of the cost function for optimization subroutine, so the more model runs, the more time needed in optimization. Figure 5 shows performances of these methods in terms of model runs they required. It is obvious that L-BFGS converges the fastest. We can conclude that of the three optimization methods L-BFGS is the best for data assimilation in STEM.

\section{Conclusions}

STEM is a multiscale atmospheric chemical transport model and has been used for air quality simulation regionally. Model Predictions can be improved by the technique of data assimilation. Data assimilation allows combining the information from both observations and STEM forward and adjoint models to obtain best estimates of the three-dimensional distribution of tracers. Reanalyzed fields can be used to initialize air quality forecast runs and have the potential to improve air quality predictions of the STEM model. Therefore, STEM is closely correlated to data assimilation. In this 
paper, we perform 4D-Var data assimilation over northeast of U.S. using the STEM model to optimize initial conditions. Both data and figures show the great improvement for simulation of STEM after data assimilation. Besides, we assess performance of three optimization methods that implement data assimilation in the STEM model. The results imply that L-BFGS best fits the STEM model of the three methods. Future work will focus on implementing second order adjoint model in STEM to provide Hessian of the cost function. In this way we can utilize some more accurate optimization methods for data assimilation.

Acknowledgemets. This work was supported by the Houston Advanced Research Center (HARC) through the award H59/2005 managed by Dr. Jay Olaguer and by the National Science Foundation through the award NSF ITR AP\&IM 0205198 managed by Dr. Frederica Darema.

\section{References}

1. Carmichael, G.R., Peters, L.K., Saylor R.D.: The STEM-II regional scale acid deposition and photochemical oxidant model - I. An overview of model development and applications. Atmospheric Environment 25A: 2077-2090, 1990.

2. Kalman, R.E.: A new approach to linear filtering and prediction problems. Trans. ASME, Ser. D: J. Basic Eng., 83:95-108, 1960.

3. Fisher, M. and Lary, D.J.: Lagrangian four-dimensional variational data assimilation of chemical species. Q.J.R. Meteorology, 121:1681-1704, 1995.

4. Khattatov, B.V., Gille, J. C., Lyjak, L.V., Brasseur, G. P., Dvortsov, V. L., Roche, A. E., and Walters, J.: Assimilation of photochemically active species and a case analysis of UARS data. Journal of Geophysical Research, 104:18715-18737, 1999.

5. Elbern, H. and Schmidt, H.: A 4D-Var chemistry data assimilation scheme for Eulerian chemistry transport modeling. Journal of Geophysical Research, 104(5):18583-18589, 1999.

6. Errera, Q. and Fonteyn, D.: Four-dimensional variational chemical assimilation of CRISTA stratospheric measurements. Journal of Geophysical Research, 106(D11):1225312265, 2001.

7. Wang, K.Y., Lary, D.J., Shallcross, D.E., Hall, S.M., and Pyle, J.A.: A review on the use of the adjoint method in four-dimensional atmospheric-chemistry data assimilation. Q.J.R. Meteorol. Soc., 127(576(Part B)):2181-2204, 2001.

8. Sandu, A., Daescu, D.N., Carmichael, G.R. and Chai, T.: Adjoint Sensitivity Analysis of Regional Air Quality Models. Journal of Computational Physics, Vol. 204: 222-252, 2005.

9. Carter, W.P.L.: Documentation of the SPARC-99 chemical mechanism for VOC reactivity assessment final report to California Air Resources Board. Technical Report. University of California at Riverside, 2000.

10. Damian, V., Sandu, A., Damian, M., Potra, F. and Carmichael, G.R.:The Kinetic PreProcessor KPP - A Software Environment for Solving Chemical Kinetic. Computers and Chemical Engineering, Vol. 26, No. 11: 1567-1579, 2002.

11. Liu, D.C. and Nocedal, J.: On the limited memory BFGS method for large-scale optimization. Math. Programming 45: 503-528, 1989.

12. Morales, J.L., and Nocedal, J.: Enriched Methods for Large-Scale Unconstrained Optimization. Computational Optimization and Applications, 21: 143-154, 2002. 